\title{
Developing viable, adjustable strategies for planning and management-A methodological approach
}

\author{
João Paulo Fernandes \\ Institute of Mediterranean Agricultural and Environmental Sciences (ICAAM), University of Évora, Núcleo da Mitra | Apartado 94 | $7006-554$ Évora, Portugal
}

\section{A R T I C L E I N F O}

\section{Keywords:}

Landscape planning and management

Strategy

PESTEL framework

SWOT

Business scorecards

Decision-making

Management culture

Organization's culture

\begin{abstract}
A B S T R A C T
The problematic of the effectiveness of the processes of decision and development of strategies and plans and ensure their effectiveness is analysed and characterized. A methodological framework using classical management instruments like PESTEL, SWOT analysis and Business Scorecards is presented, where these instruments are developed and adjusted to the requirements of the development of strategies and plans and their long-term management, implementation and adjustment. This framework aims also at enhancing the process accountability and mainly its comprehensibility, allowing its use as a basis for the strategy and plan adjustment after context or conjuncture changes, without changing its global philosophy and internal logic. An implementation conceptual example is given to illustrate the framework practical use and future developments are presented and justified.
\end{abstract}

\section{Introduction}

Decision, in any context (entrepreneurial, corporative, administrative or political), is always a more or less complex process where, although there exist a manifold number of decision support methodologies, there is still a tendency to a prevalence of more or less important intuitive or subconscious drivers (Fernandes and Guiomar, 2016).

This situation is of critical importance when developing strategies that will imply the involvement (active, passive or simply executive), of very different groups of individuals and organisations. In effect, one can observe, for example, that independently of the official discourse, the landscape planning process prevailing culture is the overwhelming arbitrary decision power of the responsible administrations. For example, the stakeholders involvement is little more than short-term consultations and, in particular, generally no importance is given to the different involved stakeholders drivers, exactly the ones that will be responsible for the plan's successful implementation.

In order to prevent and correct these problems, it is necessary a coherent, integrated strategy-development-methodology ensuring a coherent identification of all potentials and constraints (external and internal) faced by the planning or management concept to be developed. This need derives from the fact that any strategy development must balance the general objectives definition, the constraints identification and ranking, the identification of detailed objectives, the definition of implementation measures, as well as their respective success indicators and, finally, the strategic network materializing those measures and their actions and allowing the identification of critical paths and bottlenecks.

Such a methodology would allow a successive process of general objectives identification, internal and external characterization and ranking, specific objectives or targets definition based on the previous steps, and a resulting acting instrument, defining tasks, targets, achievement or performance indicators, interactions between those tasks and potential critical domains. This successive process combining well-tested instruments, allows a permanent re-evaluation of the context and criteria considered in the strategic development process and, therefore, an easier revision when those conditions change (long before they can be sensed in the assessment or performance indicators). It also allows an easier identification of the different relevant stakeholders, their motivations and willingness to act (and therefore consider strategies to fortify their motivation and rewarding factors) and particularly, the character, culture and working practices of all involved actors and organizations.

This last issue is of particular importance, because the most important factors of success of any strategy, are not the physical or economical resources or even the human qualification of those involved, but mainly the acting and decision drivers of the individuals and organisations (external or internal) involved in the strategy development and success. This corresponds, for example, on one side to the culture of the organisations (Linnenluecke, and Griffiths, 2010) and their organisational structure and daily practice. On the other side, the ability of

E-mail address: jpaf@uevora.pt. 
the acting stakeholders (e.g. normal citizens, owners, entrepreneurs, bureaucrats, politicians) to perceive the advantages of their active involvement and the higher rewarding resulting from the successful strategy implementation (Fernandes et al., 2018).

This paper aims, therefore, to propose such a strategy-developmentmethod based on the combination and innovative articulation of existing and well-tested methodologies that fail to give integrated contributions, due to normally handling only parts of the strategy development process.

The paper starts by analysing the present praxis and state of the art pointing to a situation where there are no comparative integrated methodologies but only conjunctural, unstructured approaches by each planner or organization. Then, the methodological demands of a new integrated methodology able to respond to the planning and strategy definition process are presented, and a conceptual framework proposed. Thereafter this framework is materialized based on well-proven methods that are used and integrated in an innovative and more understandable, participative-able form. To illustrate the proposed framework an application example is sketched and partly exemplified. The many issues raised by this development process and the principles behind the proposed framework are systematically presented and discussed. Finally, one tries to discuss some of the many open issues within a systematized global view of the proposed framework

\section{The current praxis and state of the art}

Strategy is the direction and scope of an organisation over the long term: which achieves advantage for the organisation through its configuration of resources within a changing environment, to meet the needs of markets and to fulfil stakeholder expectations. (Johnson et al., 2008, pp. 3).

Presently, there are many blockages in planning and management processes, either in terms of their effective application and implementation (Berke and Conroy, 2000; de Montis et al., 2014) or their effectiveness (e.g Laurian et al., 2004; Bottrill and Pressey, 2012; Milieu and IEEP, 2016; Hermoso et al., 2017) due to the way people, organizations and administrations perceive the meaning and implications of these instruments, displaying an insufficient planning culture at all society levels, including the entrepreneurial (e.g. Schäffer and Willauer, 2002). This situation occurs also at the level of the practical effectiveness of decision and control instruments like environmental impact assessment and strategic environmental assessment (Arts et al., 2012; Lobos and Partidário, 2014; Hayes et al., 2017).

This situation derives, as referred, from a lack of planning (and even, management) culture, an ability to think on the long term, an inability to develop systemic adjustable planning and management instruments and, particularly, an incapacity to involve and empower the relevant stakeholders in the planning and management process in a conscious, accountable and motivated way (e.g. Tippett et al. (2005); Maon et al., 2010; Drechsler et al., 2011; Dobrovolski et al., 2011; Soma et al., 2016; Izakovičová et al., 2018).

Another important problem is the fact that we have little idea why, how or in which aspects a plan failed, due to a general absence of assessment and evaluation procedures of each planned developments and results as well as the many individual decisions involved (e.g PintoCorreia and Azeda, 2017; Berke and Conroy, 2000; de Montis et al., 2014). Effectively, one must recognise that the systematic evaluation of the effectiveness of these instruments and practices is still very limited, independently from the existing extensive literature on evaluation indexes and methodologies (e.g. Mickwitz, 2003; Bottrill and Pressey, 2012). Nevertheless, some key aspects can be identified as being predominantly common to these processes:

1 The strategy and plan development is predominantly arbitrary being normally developed by a small group of technicians and eventually decision-makers, with little or no assessment of the effective needs and expectation of the society, and involved organizations or stakeholders;

2 The core of the strategy, plan and practical management is not assumed and conducted by the management team, being delegated and many times neglected, or arbitrarily changed by it or by sectorial coordinators.

3 The organization's management and the main chain of responsibility and decision-making predominantly lacks an integrated coherent planning and management culture, namely in terms of an accounting and empowerment praxis defining chains of responsibility and information interchange as, for example, demanded by ISO 9000.

4 Most strategies, plans, management actions and decisions ignore that the ability to implement them, does not lay on the decision maker, but on common citizens, workers and other stakeholders who end up predominantly ignored and even disregarded in the planning and decision-making process.

5 The strategy, plan or management criteria and development processes are normally rigid lacking their driving criteria and philosophy, resulting in an inability to adjust to context and conjuncture changes and to be adapted without changes in its internal logic.

This diagnosis, common in enterprises, organizations of all nature and administrations is still reinforced by a predominant culture and practice of arrogance, concealment, prepotency, confrontation, and contempt towards workers, communities as well as individual values and rights (e.g. Sandman, 1993).

This results in opaque, ineffective, maladjusted instruments, predominantly divorced from the ones who will implement them (primary stakeholders, mostly). Ignoring the basic need to ensure any restriction of freedom or right (e.g. property) will only be accepted and implemented if it is perceived as fair, adequately and rewardingly compensated.

This diagnostic implies the need for easy to implement working frameworks (using well-tested instruments and methodologies) that are easy to understand and handle, according to context and conjuncture modifications, accountable and able to permanently identify critical paths and bottlenecks in order to handle them in a focused and contextualised way.

Such a framework must include instruments allowing an effective and detailed accountability, building the basis for an assumed stakeholder's involvement and practically implementing the development of new social and organizational cultures indispensable for the success of the strategy, it's planning development and implementation.

\section{Methodological framework - conceptual requirements}

The development of strategic instruments for planning and decision making instead of a linear process is a dynamic, integrated, interactive process. It involves a diagnostic, a definition of objectives (not targets because these are rigid and unsusceptible to contextual adjustments) and a set of instruments to identify and define the ways to achieve those objectives, allowing, at the same time, their permanent re-evaluation and adjustment. All these instruments must be clear, objective and reflect the interests, involvement and accountable empowerment of all involved stakeholders.

Therefore, one has to approach and solve several successive or simultaneous and interactive tasks involving characterization, evaluation, assessment and validation processes in a universe of permanent interaction with stakeholders and decision-makers.

First, one must define the global objectives and make the diagnostic of the existing conditions (internal and external) which will determine the ability to achieve those goals. These two processes must be interactive: the diagnostic of the present situation and its predictable evolution, combined with the evaluation of the existing resources, is critical. This becomes necessary since the eventual need to exclude some 
objectives due lack of resources or conditions should imply a planned ranking of their relevance in the strategy and plan development.

The diagnostic must be performed considering not only the external conditions (favourable or constraining) but also, and with particular attention, the internal conditions - focused in the organization's, administration's or society's culture, which will determine their ability and willingness to perform the required tasks. Also of utmost importance is the availability of effective accountability systems that guarantees all stakeholders, now informed and effectively participating (empowered) in the implementation process, will willingly involve and foster themselves throughout that process.

In tandem, the diagnostic must also rank the different conditioning factors and agents according to their relevance and ability to affect the strategy implementation process, whilst identifying which ones are manageable and which are not (and eventually act as potential risk factors for the implementation process feasibility).

When the diagnostic and global objectives are defined, and the potentials and constraints ranked and characterized, it is the moment to define sectorial objectives, corresponding to the different domains in which the global objectives are materialised. These sectorial objectives must be tangible, easy to characterize, but, most of all, must focus and address each of the problems and potentials identified in the diagnostic phase.

Their implementation must be assessed by instruments identifying the involved key strategic issues - these key performance indexes that must be used to evaluate the degree of achievement of the specific tasks, and define minimal levels of success determining, if not achieved, the need to reformulate the strategy or the reassessment of the performance of the involved organizations and stakeholders.

Based on all this information and assessment instruments it will then be possible to design the global strategy, where all actions and interactions in all relevant domains are identified, allowing a systemsdynamic approach able to better characterize those actions and interactions, as well as the necessary instruments and controls.

This succession of steps, although not innovative (see for example Bryson and Alston, 2004), are only very partially used in most strategy and planning development as well as management processes, both in the entrepreneurial and even less in the public sectors (landscape and other development plans), mostly due to the deficient integration of the available working instruments and frameworks. An innovative approach is therefore required, aiming the creative integration of tested methods allowing their use as assessment and accountability instruments but also the introduction of new perspectives of application of some of them, focusing on the need to respond to any major constraints in strategy and planning construction as well as implementation: socioeconomic and organizational culture.

\section{Proposed framework}

Bryson (2018) stresses, in the context of the development of the strategic development cycle, the need to simultaneously consider and integrate the exterior and interior environments of any organization, administration or society, in order to be successful. This consideration derives from the obvious acknowledgement that the development of any strategy depends not only on the external development context but also the characteristics, structure, way of working and global culture of the ones that will conceive and, particularly, develop that strategy.

\subsection{Diagnostic: potentiating the PESTEL and SWOT frameworks}

In order to develop the diagnostic phase, it is important to use a well-known and experienced framework conceived to analyse and monitor the macro-environmental (external environment) factors that have an impact on an organisation. More important still is to ensure that such a framework is easily adjustable to the targets of simultaneously developing a methodological replicable and coherent external and internal analysis, but also allow the ranking of the identified characteristics as well as the distinction between manageable and nonmanageable ones. The selected framework is PESTEL (Gillespie, 2007).

PESTEL is an acronym for the following factors affecting any organised structure or even individuals:

$$
\begin{aligned}
& \text { - P - Political } \\
& \text { - E - Economic } \\
& \text { - S - Social } \\
& \text { - T - Technological } \\
& \text { - E - Environmental } \\
& \text { - L - Legal }
\end{aligned}
$$

While there are manifold references on the possible variables that can be considered when analysing external environments, its use for internal environments, because seldom used, must be better characterized and exemplified:

Political - the structure, internal relations of power, decision, information, accountability within any organization (or society) are critical in defining the way that organization is able to respond to particular tasks, changes or challenges (e.g. Hellriegel et al., 1998). ISO 9000 , only to give an example, stresses as basic conditions for an effective organization management in terms of quality, the importance of a clear organization's structure, definition and attribution of tasks, responsibilities and ability to decide, together with an informed and committed administration.

Economic - the financial conditions of any organization, not only in terms of the different forms of capital and assets, but also external image (confidence and reliability), are critical in determining its ability to cope with the challenges of any strategy development. These conditions have to ensure the feasibility of the necessary investments [including the costs associated with non-manageable variables (like, for example, external arbitrary decision makers, payment delays, internal organisation, work force or decision bottlenecks)] as well as the short and long-term maintenance costs. Another important aspect to be taken into consideration is the economic rationality of any action or strategic option - if one will only have costs without revenue, the option is not viable.

Social - here lies one of the critical internal issues, because it refers to the individual and institutional culture, paradigms, habits, values, convictions and general decision plus behavioural drivers. These are variables very difficult to manage and involve the entire organization/ society. Keywords are accountability, empowerment, confidence, openness, interaction, attention, all integrated and aggregating the global organizational structure, behaviour and posture (Goodland, 2002). The lack of these conditions can undermine success factors like workforce committed involvement, prevention of internal or external conflicts, secure reputation, confidence and permanently be able to predict, identify, prevent and adapt to new internal and external factors.

Technological - the ability to use, adapt and develop available technologies, to adjust to new developments and to permanently ensure the technical upgrade of all involved in the organization is critical. In effect, an organization needs to be able to permanently upgrade the technological ability of its entire workforce, not only in terms of those that use the technology but also those who manage its use. The ability to ensure internal horizontal and vertical interactive flows of communication enabling the effective consideration of improvement, suggestions and to effectively and quickly clarifying the why and how of any technological change, are essential to ensure the ability to adapt, evolve and innovate.

Environmental - there are two central issues for any organization when handling environmental issues: cost reduction (both in terms of internal and external costs), therefore, resource use optimisation and risk prevention (either physical or corporate). The other central issue is cost-benefit analysis not only in direct and indirect immediate and 
comprehensible economical terms, but also a comprehensive assessment of all costs and benefices, including social costs. This means that environmental issues must be considered as any other decision factor and environmental assessment must be naturally incorporated in the general organization's feasibility assessment and investment evaluation.

Legal - any society, organization or administration is subject to legal frameworks that constrain and regulate the way they must function. Associated, but equally important, are the internal objective or subjective rules, that derive from the society and organizational culture. Many of these rules are not formally expressed, but are as determinant (or even more) as any legal imposition (e.g. Abreu-Santos et al., 2017). Also important to take into consideration in this domain, are all ethical questions that respect the way internal relations work within the organization, but also, and critically important, is the way the stakeholder's interests and concerns are taken into account.

Taking into account that the result of the PESTEL analysis are normally lists of factors aggregated according to each thematic, it is to note this will always lack a ranking of their importance in the analysis context as well as the reference to their manageability. This information must be incorporated in this reviewed framework (both in the external and internal diagnostic). Therefore, one must consider the need to perform parallel interactive analysis exploring external and internal factors and different relevance ranks of the considered factors.

One last remark concerning this stage of the diagnostic is the need for detail. Each identified issue must be referred not in general terms, but referencing in detail it's relevace4 in each domain. It can even happen that two aspects of the same variable or domain can have opposite influences that must be, therefore, clearly distinguished.

These aspects are very important in the next step of the diagnostic: the SWOT analysis.

Again, it is a decade old well-known and used method, which can, if inadequately used, be of irrelevant interest to the strategy and planning development process (Hill and Westbrook, 1997). Nevertheless, if the PESTEL framework is correctly used and in the detailed and qualitative way (including rankings and manageability evaluation) as previously described, it is possible to develop both integrated and thematic SWOT charts, identifying not only the critical issues to be handled, but also their relative importance and manageability (developing, for example, concepts presented by Aithal, 2017).

The articulation between PESTEL and SWAT is made through identifying all existing variable, using the PESTEL framework:; the role of each variable, external or internal, as well as their ranking in terms of relevance for the purpose of the strategy development. As a result, in the same way that different PESTEL tables are developed, also corresponding different SWOT analysis are made complementing each other according to the character of the considered factors (determined by PESTEL).

\subsection{General objective definition}

When considering this target, one has to be aware that there are two main perspectives or approaches: top-down or bottom-up. Independently from the specific adoption of any one of these, Gregory et al. (2012) refer several practical methods of strategic objective definition. The most common is the classical expert based process, where the decision is taken by an individual or panel of experts based on models and simulations. This approach generally lacks a validation procedure, omits critical evaluation such as cost-benefit and economic viability and has a clear arbitrary character. In another approach, the consensus-based decision-making, is only possible on the frame of a restricted group (limiting the consideration of minority perspectives) and is normally developed before a full diagnostic and analysis of the subject is available - this limits the consideration of many factors such as uncertainty factors, giving little attention to the stakeholders perceptions and, particularly, involving a limited sustained bargaining process that does not reflect the necessary trade-offs and contractualization processes that only an adequate knowledge and involvement of all relevant actors allow. Finally, economics and multicriteria analysis, can be biased due to the way the criteria and involved participants are selected, but remains one of the best basis for developing more consensual decisions in a multiple agents context.

Considering that "the essence of good decision-making lies in understanding the problem, gaining insight into what matters to people, and then generating responsive alternatives" (Gregory et al., 2012, pp 5 ), these authors propose as a more efficient alternative, the structured decision-making (SDM). It consists in "the collaborative and facilitated application of multiple objective decision making and group deliberation methods to environmental management and public policy problems. It combines analytical methods drawn from decision analysis and applied ecology with insights into human judgment and behaviour from cognitive psychology, group dynamics, and negotiation theory and practice. The primary purpose of an SDM process is to aid and inform decision makers, rather than to prescribe a preferred solution" (Gregory et al., 2012, pp 6). It consists in a permanent interactive process, based on an adequate diagnostic of the departing situation, the assessment of different alternatives and associated evaluation. This must be complemented by a permanent assessment of the process and context evolution, allowing a consistent and timely adjustment of the objectives to new realities and constraints (highlighting the importance of the possibility to permanently update the PESTEL and SWOT results).

This methodology is based on the answers given to the following questions (idem pp. 7).

1 What is the context for (scope and bounds of) the decision?

2 What objectives and performance measures will be used to identify and evaluate the alternatives?

3 What are the alternative actions or strategies under consideration? 4 What are the expected consequences of these actions or strategies? 5 What are the important uncertainties and how do they affect management choices?

6 What are the key trade-offs among consequences?

7 How can the decision be implemented in a way that promotes learning over time and provides opportunities to revise management actions based on what is learned?

This is achieved through completion of the following steps, represented in Fig. 1, combined with the diagnostic and assessment tools provided be PESTEL and SWOT as proposed. This implies combining the referred bottom-up and top-down approaches trough an effective interactive process of information exchange throughout the management structure, where all strategic issues and constraints are widely considered within the organization.

Fig. 1 clearly illustrates the interactivity of the decision-making processes and the need for a permanent update of the considered data and criteria, validating each step whilst ensuring an effective contribution to the next one. Therefore, instead of a linear process, this is a dynamic self-assessed and adaptable process able to permanently adjust itself to conjunctural changes.

\subsection{Integration and definition of thematic and specific objectives and partial strategies}

Based on the different SWOT analysis resulting from the PESTEL diagnostic processes, a set of dully ranked issues (positive or negative, internal or external, manageable or not) are identified, allowing the application of another integration methodology aimed to be the base for the development of a global integrated strategy: the Balanced Scorecard (BSC) (Kaplan and Norton, 2001). It aims primarily at allowing the consideration of non-metrics performance indicators in a global perspective, in order to build aimed action plans to achieve each particular goal in a set of strategic domains and allowing their 


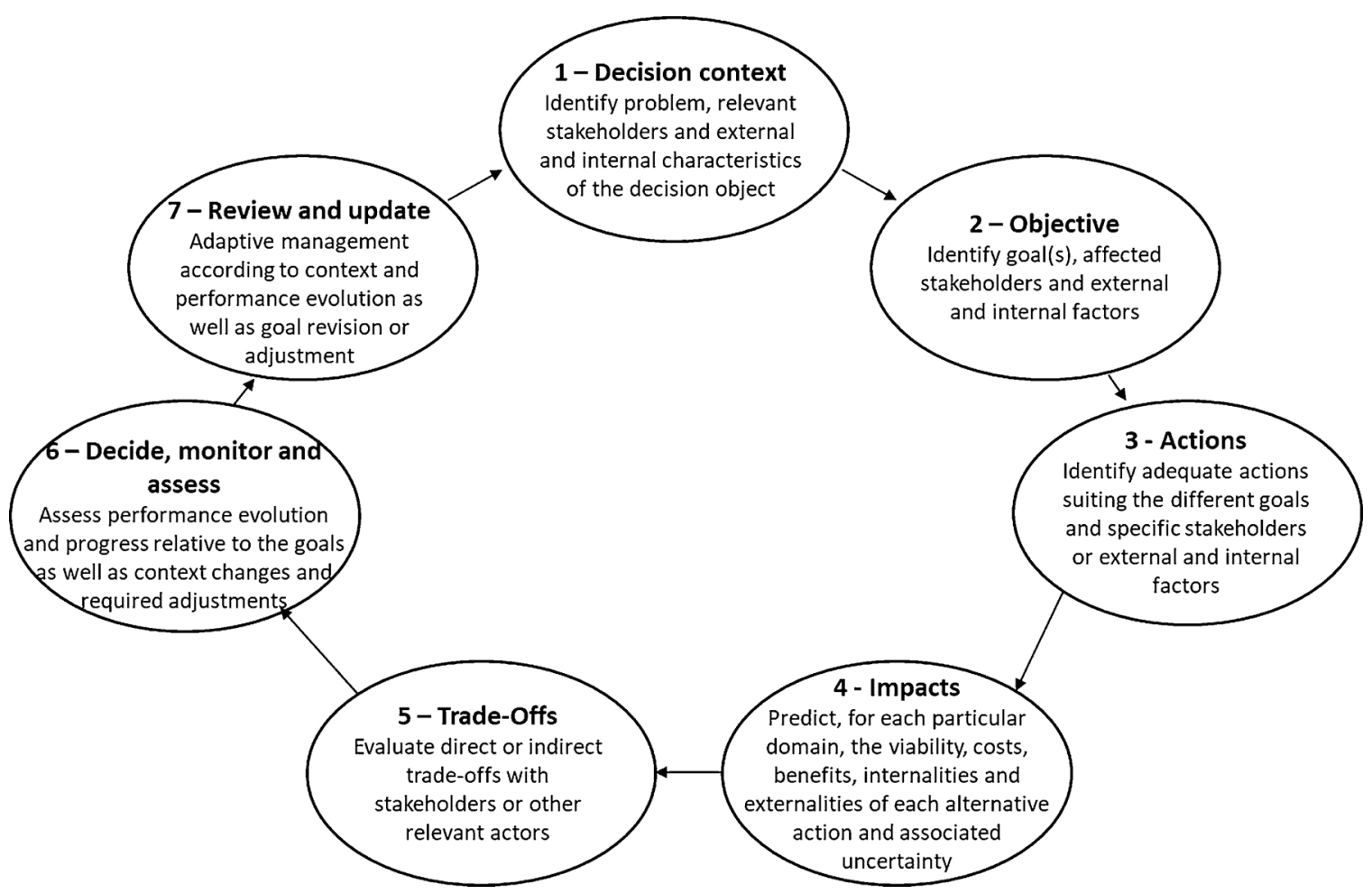

Fig. 1. Steps in structured decision-making (adapted from Moore, and Runge, 2012).

integration in a global strategy. The use of BSC allows turning the SWOT analysis into strategic approaches.

The BSC methodology has proven results in the entrepreneurial management for which it was created, but is now being more often introduced for environmental as well as planning and landscape management (e.g. Kaplan, 2009 and Kaplan, 2012; Niven, 2010; Al-Zwyalif, 2017).

This method follows the general guidelines proposed by Kaplan and Norton in 1992 starting with the elaboration of a set of BSC for each relevant perspective (originally stakeholder/customer, internal processes, learning and growth and financial/investments), but presently also for two new perspectives such as employee satisfaction and environmental community (Kaplan et al., 2004; Parmenter, 2015).

These six perspectives and associated indicators are considered as the best adapted to support the organizations vision and strategies, and give a better structure to the strategic management plan development. Nevertheless, for the present framework several adjustments must be made (Fig. 2). The customer perspective must be enhanced to the citizen and stakeholders perspective, given that citizens, are the key "customers" of policies, plans and landscape management strategies, together with other stakeholders, like enterprises, administrations and other organizations (profitable or non-profit). The environmental / community perspective is more adequately addressed as a sustainable perspective ("sustainability perspective"), because it builds the basis for the entire strategic concept by setting its practical and conceptual frames. Finally, the employee satisfaction perspective is better described in this context as the "active actors satisfaction perspective" due to the diversity of their character (not only individuals but also organizations).

The introduction of these two new perspectives follows the same logic present by Kaplan and Norton (2001) starting with the sustainability perspective as the conceptual and framework definer, followed by the actors satisfaction perspective. This is relevant because these build the executive bridge between the sustainability conceptual philosophy and the specific needs of the strategy and plan to be implemented. In effect if these actors ("doers") are not fully aware, involved and empowered, they will not adequately fulfil the necessary tasks involved in the strategy implementation (Fernandes and Guiomar, 2016).

The BSC approach identifies, for each perspective, a set of domains (Key Success Factors) that are characterised by not necessarily quantitative metrics (Key Performance Indicators - KPI). These allow the definition of performance target levels that require, in order to be attained, the definition of specific action plans that will build the basis for the development of the organization's strategy (Kaplan, 2009).

In this sense, based on the PESTEL / SWOT analysis, general targets for each perspective can be built, and the different issues identified as relevant in the diagnostic process will then determine the base for the definition of the different key success factors. This process can be developed in different plans of consideration: if the systems complexity demands intermediary steps focused, for example, in the PESTEL six factors or domains, the systems are later integrated according to the most relevant issues resulting from this analysis.

This intercalary subdivision is important because one of the main targets of this framework is to ensure that the entire strategy development process and all considered variables and criteria are clearly displayed and can be easily understood. This is not only important in order to understand the development process, but also to allow its targeted revision by all involved stakeholders whilst safekeeping the initially considered premises.

BSC can be misused when managers focus only in fullfiling KPI, independently from their interactions and interdependencies and, particularly, in terms of the global fulfillment in all perspectives, and not only in one considered more relevant (as the financial). This attitude of being unable to understand that the sustainable development of one organization is not related to the circumstantial fulfillment of some KPI, but to the overall long-term success of the different perspectives in achieving the global strategic objective, is a clear obstacle to a comprehensive and successful management.

To avoid this, we must focus our attention on the Summary Action Plans for each Key Strategic Factor and ensure that in their definition, all interactions between all relevant factors, from all perspectives, are 


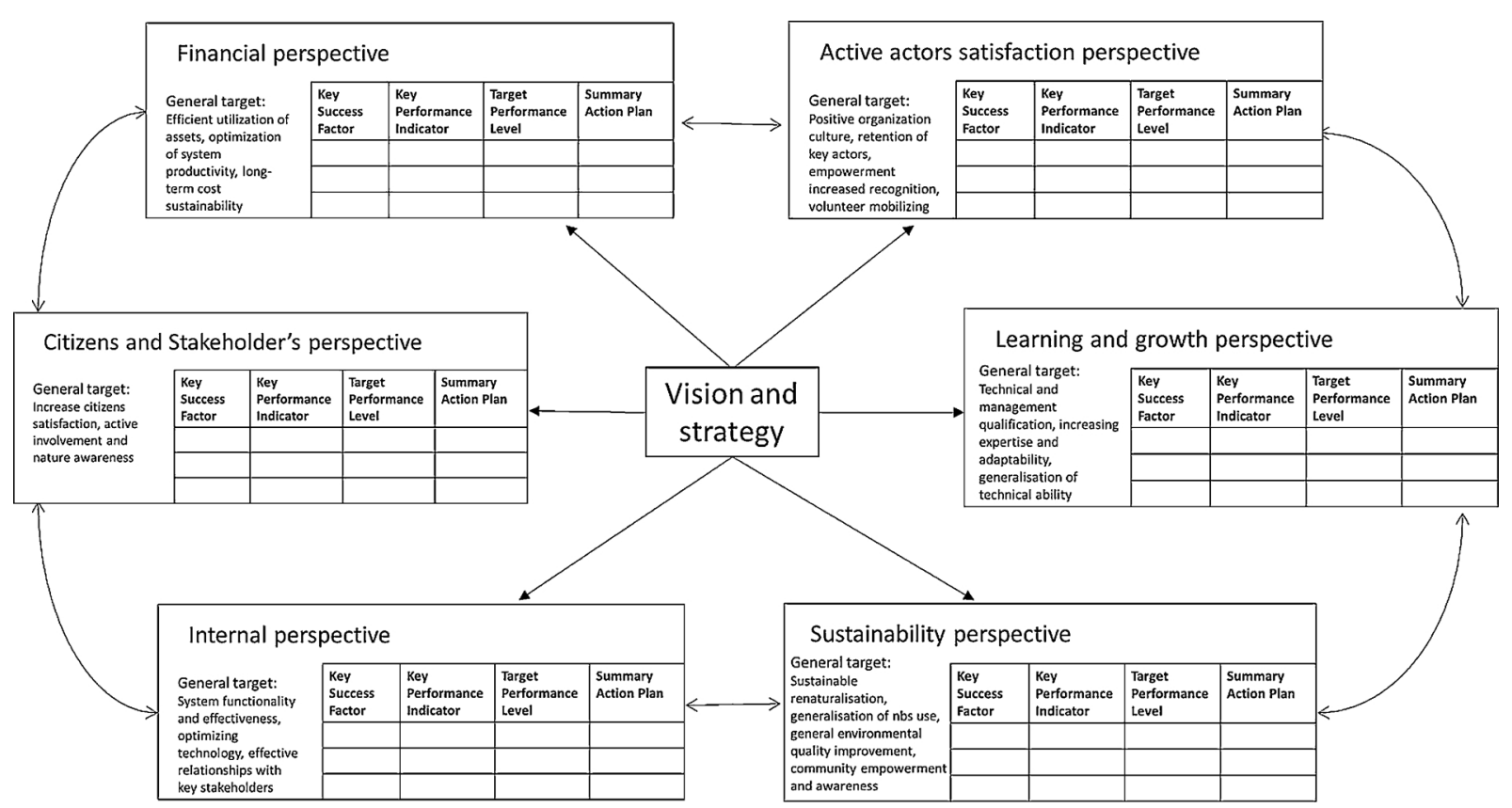

Fig. 2. Translating vision and strategy - six working perspectives for a conceptual example (adapted from Parmenter, 2015).

taken into consideration. This is an often-misunderstood problem while using BSC, where each perspective, as well as each strategic factor and correspondent strategy action, are seen as independent and having only a single linear relationship.

\subsection{Building and operating a strategy and the corresponding planning process}

The final product of the BSC methodology is the construction of strategy maps, which are diagrams where, for all perspectives, the key strategy factors are identified, and their interdependence in the frame of the global and sectorial objectives are displayed showing the way strategic objectives can be attained through a strategic cause and effect relationship. These Maps build a graphical link between the BSC and the conceptualization and execution of a working strategy.

The Strategy Map is more than a simple graphic representation of the actions and relations between the different perspectives and success factors, aiming at clarifying the full scope of interactions that the strategy implementation involves. This can imply their subdivision (or detailed division) in sub-strategies for particular scenarios of more complexity.

Taking this into consideration, one will then be able to implement the strategy execution process, either in terms of planning development or management implementation (Fig. 3).

This process, focused on the strategy map, the different action plans and their interactions is, as the structured decision-making process, a permanently interactive process able to adjust to contextual changes without the need to change the working philosophy. This is possible because the way the strategy development process was conducted allows pinpointing the specific changes and adjusting without the need of a full redesign or a even a conjunctural arbitrary change of strategy. Also particular relevant, is the importance given to the permanent process assessment and monitoring, in order to permanently adjust the organization to the evolution of the strategy and the internal and external reality.

Fig. 4 summarizes graphically the entire proposed framework where is possible to verify the articulation between PESTEL and SWOT and their integration in order not only to support the definition of the global and sectorial objectives as well as different BSC analysis and their later application on the elaboration of the strategy plan. All steps are adjusted via an external and internal validation by all involved stakeholders.

\section{Implementing the framework: a conceptual example}

It is not our aim to present a fully developed example of the proposed framework due to its complexity and dimension, incompatible with the scope of this paper. Therefore, only sub thematic examples of specific innovative approaches will be presented to illustrate the main characteristics and advantages of this integrated methodology.

The general target of ecological restoration in urban environments can be considered and materialised according to many perspectives and strategies, depending on the existing conditions: natural, social and infrastructural context, financial means and societal acceptance and involvement. Also, the available techniques and philosophical intervention perspectives are manifold, from the simple reintroduction of nature in the urban environment to the fully restoration of habitats and communities.

Therefore, the definition of the global objective for a particular case is always a specific unique task. It must be developed and defined according to the diagnostic of its particular context and predictable evolution patterns.

The external PESTEL analysis will have to consider safety issues. Safety issues, many of the possible intervention sites can be sites with particular risk constraints (like steep or unstable slopes or cliffs or where interventions can interact with hydrological and hydraulic processes and associated flood risks). In addition, legal constraints and propriety issues must be identified, together with the different owners or the ones responsible for the affected infrastructures. The list is necessarily long but two particular items have special relevance: societal posture in relation to these interventions; the different postures of the involved administrations or administration sectors. While the first issue is a manageable variable, since the involved stakeholders are integrated in the program, the second is a non-manageable variable because it deals with power issues (expression of the individual relevance of the sector or administration director for example) in domains where arbitrariness can be of high relevance, although not clearly and openly explicit.

These last examples are not commonly considered in these analyses and can be so relevant or even more relevant than, for example, legal 


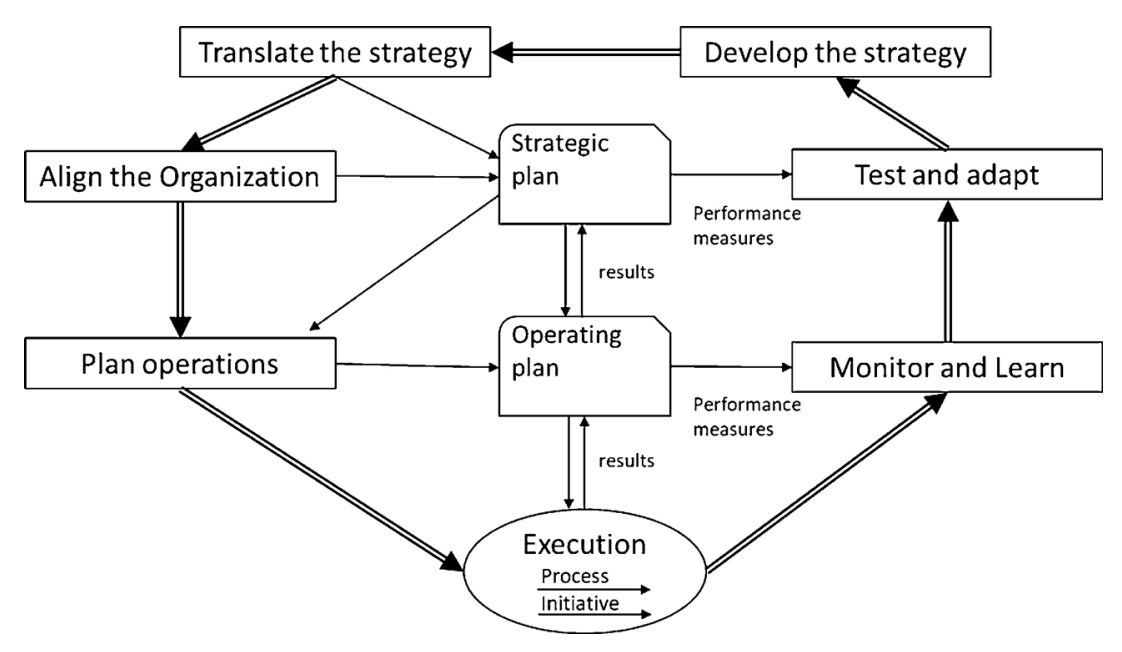

Fig. 3. A closed loop Management System for Strategy Execution (adapted from Kaplan, 2009).

regulations or financial constraints (see, for example, Sandman, 1993).

The internal PESTEL analysis deals with those who directly or indirectly will implement the strategy. In this universe, one has, for example, to consider all financial constraints: investment capital (including the eventual costs of license delays, technical problems or citizen contestation), maintenance costs, cost-benefit analysis, internalities and costs of internalization of external costs. Also critical, are the organization hierarchies and culture: the way the different tasks are assigned and coordinated, as well as the power of decision attribution, the way responsibility and accountability are implemented and assessed, the effective involvement of the different actors, administrations, organizations and (formal and informal) power structures. Finally, only to mention some of the most critical internal factors, are the informed commitment of all involved deciders and managers (present or foreseeable) as well as all relevant individuals in the structure chain of power, intermediary decision and information exchange.
These are all simple examples of domains where too often, projects, plans and programs fail, because they were unable, from inception, to understand that the stakeholder's involvement is both an external and an internal factor. Power management (mainly the informal and arbitrary) is also a critical issue when developing long-term strategies and plans, this is why one has to create consensus to ensure the strategy implementation and the plan's continuity.

One practical example: the Zurich brook/small stream concept (BachKonzept), circa 25 years, is channelling small streams to a near to natural form, integrating them in the city structure and creating new living and leisure spaces. This project and its implementation meant the total rearrangement of the city's drainage and wastewater treatment structure (with important savings due to reduction of the volume to be treated), a general commitment of all parties involved, to ensure the long term development of the concept, and an effective and growingly active involvement of the citizens and other users and managers of the urban space. This achievement was only possible through a long-term

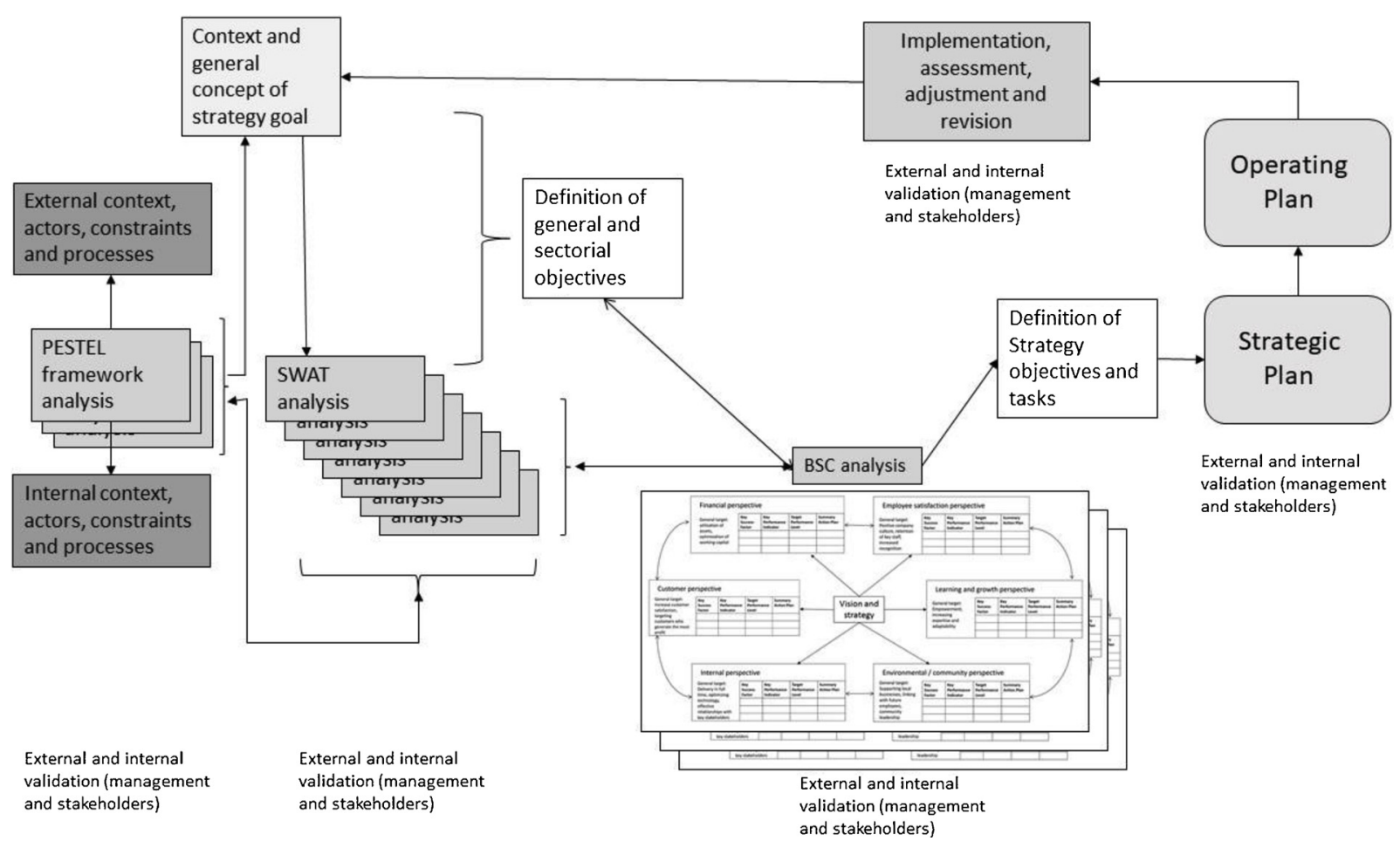

Fig. 4. Global schematic layout of the proposed framework. 
Table 1

Example of a Business Score Card for the Citizen Perspective in the frame of the conceptual application to ecological restoration in urban environments with the exemplification for the Success Factor "Involvement" of the interconnections with the other BSC framework perspectives.

General targets: Increase citizens satisfaction, active involvement and nature awareness

\begin{tabular}{|c|c|c|c|}
\hline Key Success Factor & Key Performance Indicator & Target Performance Level & Summary Action Plan \\
\hline $\begin{array}{c}\text { Perception and } \\
\text { awareness }\end{array}$ & $\begin{array}{l}\text { Percentage of citizens aware of } \\
\text { the goals and benefices }\end{array}$ & $\begin{array}{l}\text { Ensure a minimum } 15 \% \text { yearly growth } \\
\text { ratio }\end{array}$ & $\begin{array}{l}\text { Launch previous to any intervention a personalised information campaign. } \\
\text { Involve the direct stakeholders on the final conceptualization. Involve the } \\
\text { direct stakeholders in the monitoring process. Develop a permanent } \\
\text { targeted information system on the intervention results. Implement an } \\
\text { effective accountable quality inspection system }\end{array}$ \\
\hline \multirow[t]{2}{*}{ Involvement } & $\begin{array}{l}\text { Users of renaturalized leisure } \\
\text { areas }\end{array}$ & Increase of $5 \%$ each trimester & $\begin{array}{l}\text { Launch information initiatives for particular target groups: school children, } \\
\text { birdwatchers, seniors. Make regular information updates on each } \\
\text { intervention evolution and new livingness experiences. Promote the } \\
\text { creation of caretakers organizations, providing formation and tools and } \\
\text { efective accountable management empowerement. Promote interchange of } \\
\text { experiences between diferent associations and users of different } \\
\text { interventions. Ensure the leisure character of the interventions. Maintain } \\
\text { regular updated information and direct experience events. }\end{array}$ \\
\hline & $\begin{array}{l}\text { Number of voluntary } \\
\text { caretakers }\end{array}$ & $\begin{array}{l}\text { Ensure a minimum } 10 \% \text { yearly growth } \\
\text { ratio }\end{array}$ & $\begin{array}{l}\text { Articulate with "Active actors" trough the enlargement of their universe to that of } \\
\text { the involved or motivated citizens; with "Sustainability" in terms efective of } \\
\text { rewarded developement of circular economy instruments and measures (e.g. } \\
\text { reduction of wastwater treatment costs or of air and water quality trough nature } \\
\text { based solutions), with "Learning and growth" trough a comprehensive and } \\
\text { extensive environmental education program together with technical formation } \\
\text { initiatives on the different technical domains associated with the implementation } \\
\text { of nature based solutions, with "Financial" trough an open accountability of the } \\
\text { program costs, investments and benefits and "Internal" trough practical } \\
\text { empowerment mechanisms. }\end{array}$ \\
\hline $\begin{array}{l}\text { Claims for project } \\
\text { expansion }\end{array}$ & $\begin{array}{l}\text { Number of new intervention } \\
\text { proposals } \\
\text { Number of new voluntary } \\
\text { caretakers organizations }\end{array}$ & $\begin{array}{l}\text { Ensure a minimum } 5 \% \text { yearly growth } \\
\text { ratio } \\
\text { Ensure a minimum } 5 \% \text { yearly growth } \\
\text { ratio of voluntary caretakers } \\
\text { organizations }\end{array}$ & $\begin{array}{l}\text { Promote a permanent city-wide information campaign of the interventions, } \\
\text { their results and inviting the citizens to experience them. Divulge the } \\
\text { expansion possibilities. Promote citizens involvement in construction and } \\
\text { maintenance trough voluntary caretakers organizations, reducing costs and } \\
\text { allowing a wider intervention ability. }\end{array}$ \\
\hline Willingness to pay & $\begin{array}{l}\text { Value of voluntary } \\
\text { contributions } \\
\text { Value of private land made } \\
\text { avallable }\end{array}$ & $\begin{array}{l}\text { Ensure a minimum } 5 \% \text { yearly growth } \\
\text { ratio } \\
\text { Ensure a minimum } 5 \% \text { yearly growth } \\
\text { ratio }\end{array}$ & $\begin{array}{l}\text { Promote within the voluntary caretakers organizations of funding leagues } \\
\text { of "friends of the renaturalization". Articulate with schools and universities } \\
\text { the inclusion of practical planning and construction activities on their } \\
\text { curricula. Promote planning and construction actions with a professional } \\
\text { certification. Give fiscal retributions to land donations or long term } \\
\text { concession. }\end{array}$ \\
\hline
\end{tabular}

strategy, involving and articulating all internal and external factors and actors.

The process of integration of this diagnostic and these complex issues in the conceptualization of the global objective involve different phases of stakeholders engagement (not all, but selected groups according to each issue in question). Particularly, it is necessary to dispose of an effective instrument of characterization and evaluation of the current situation (diagnostic) and the proposed alternatives. This instrument must be able to sustain cost-benefit and other analysis, in order to insure a permanent general comprehensive source of information and perception of all alternatives, possible trade-offs and be an analytical basis for the analysis and comparison of new proposals and alternatives (Fernandes et al., 2017).

Then, the implementation of the iterative process of structured decision-making will be possible.

Following this first stage of strategy conception and plan development, it is necessary to implement the complex SWOT analysis. This analysis must be carefully conducted because it must not be a set of generalised statements of different diagnosed domains under the light of the global objective, instead it has to detail and further rank the critical issues (not only from their apparent relevance but according to their perceived importance). In this way, this SWOT analysis will, therefore, build the basis for the definition of the sectorial objectives and targets as well as the Key Strategic Issues and derived Key Success Factors.

Going back to the suggested example, one can refer the issue of hydrological and hydraulic management. This is a domain where one is confronted with the need to evaluate and manage two complementary interdependent realities: the streamline and the watershed.
This situation implies two alternative approaches: first, handling each watershed as a separate planning entity; secondly, opting for more general Key Strategic Issues, eventually complemented by particular aspects exclusive of particular watersheds. The second approach is, according to the framework perspective, the best adjusted, because it ensures the coherence of the strategic development process.

At this stage, all the basis for the definition, either of the thematic strategic priorities and objectives, or the Key Strategic Issues, are available. The chalenge now is to ensure the consistence within the framework of the BSC approach.

The solution to this challenge starts with a careful and consistent definition of sectorial objectives (or set of objectives) for each one of the six perspectives, as well as which are the corresponding Key Success Factors and adequate KPI's. Next comes the Summary Action Plan, which must, for every Key Success Factor, define a set of proposed measures, stressing the interrelations and interdependences, with similar ones from other Action Plans as exemplified in Table 1.

Fig. 5 illustrates the global strategy map as well as the derived detailed map for the success factor exemplified in Table 1. It is therefore visible the way BSC materialize successively in actions and how these ones are justified, allowing the revision of individual actions without disrupting the global logic of the strategy.

\section{Future developments}

As expressed in the beginning of this paper this is a conceptual proposal for a methodological framework for strategy development, within the planning and landscape management context.

This methodology is materialising in the frame of the "Várzea de 


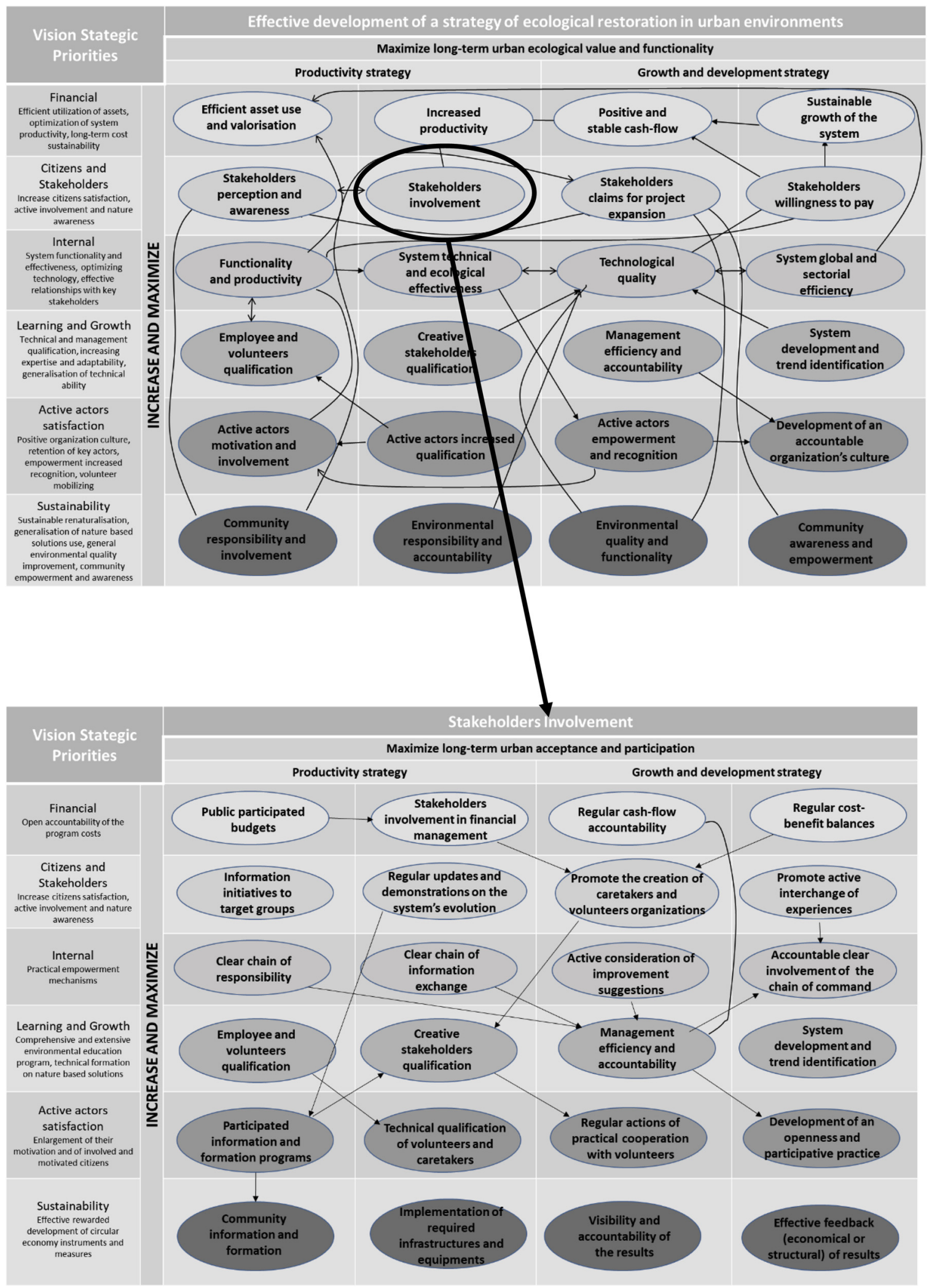

Fig. 5. Example of a global strategy map and a detailed one for one particular action or target.

Loures Project" (implementing the results from the INTERREG SUDOE NATURBA project). It aims at integrate densely urban areas, several thousand agriculture hectares to protect from catastrophic floods and simultaneously integrate in the urban living space (maintaining its production ability), and $30 \mathrm{~km}$ of waterways to renaturalize as well as creating a large urban park and connecting all these areas through a functional network of roads, paths and bicycle treks.

Due to the difficulties faced with the traditional strategy definition and planning techniques, and the interdisciplinarity of such a project, new methodological frameworks had to be developed as previously 
referred.

These new frameworks try to articulate very extensively tested methodologies from the entrepreneurial sector and combine them in a sole framework allowing a comprehensive process of strategy development, where all data and criteria are displayed and the development of the decision process is clear. This allows not only for accountable strategy and planning development processes, but also their easy reassessment and adaptation in the frame of a contextual or conjunctural change.

There are many practical issues that must be further developed in terms of the articulation between the different adopted methodologies, in order to ensure that their results are compatible amongst themselves.

The multidimensional character that such a complex process implies must also be conceived and tested in terms of its operability.

Nevertheless, the presented framework aims at building a comprehensive approach to a process that currently lacks integration, by allowing arbitrary decisions and failing in terms of accountability and ability to adapt to changing circumstances. Particularly, it aims for more transparent and responsible processes, requiring adequate implementation, with full engagement of all stakeholders, which is only possible if the development and decision processes are clear and understandable. For example, in our case study the management of the conflicts between farmers, urban citizens, wastewater treatment plant operatives, etc. was made easier through the successive participation, validation and correction steps that the framework allows (Fig. 4).

Therefore, it is important to deepen the abilities to characterize the internal factors (particularly those associated with cultural, paradigmatic, behavioural or traditional aspects) and the way they interact with the functioning of organisations and societies. Also critical, is recognising all structural factors involved (the way an organization works, information and responsibilities flow and are attributed and accounted for). The economical perspectives are also often misunderstood in the sense that they can be restricted only to financial issues, misleading the importance of the long term or the several dimensions of the cost-benefit balance, ignoring the importance of non-commodity outputs and the real dimension and character of gain associated with any action or decision.

Another important development is the way the ranked internal and external results are integrated in the SWOT analysis and can be aggregated in sets of sectorial objectives to be integrated in the different BSC's. The SWOT analysis must, therefore, be perceived not as a result, but as an integrated valuation of the PESTEL results conducting to the BSC's synthetic aggregation.

The use of the BSC framework presents the most challenges since it relies particularly in already developed integrated strategies. This means it will always be necessary to crosscheck multidimensional levels of analysis as well as "on-the-go" adjustment of criteria, actions and decisions - a domain one must handle with strategy maps and instruments, like system dynamics, to cope with their multidimensionality.

These are only some reflections on the multiple issues that this integrated methodology arises.

\section{Acknowledgments}

I want to thank the Loures municipality for my participation in the NATURBA project. I want also to thank the contributions of Prof. BeloMoreira, an anonymous reviewer, Maria Fernandes, Nuno Guiomar and Ana Fernandes that contributed to enrich and correct this paper.

\section{References}

Abreu-Santos, I., Vasconcelos, L., Pires, I., 2017. Learning from risk: lessons from L'Aquila and Japan/Aprender del riesgo: lecciones de L’Aquila y Japón. Psyecology 8 (1), 107-147.

Aithal, P.S., 2017. A critical study on various frameworks used to analyse international business and its environment. Int. J. Appl. Eng. Manage. Lett. 1 (No. 2), 78-97. https://doi.org/10. 5281/zenodo. 1053578 .
Al-Zwyalif, I.M., 2017. Using a balanced scorecard approach to measure environmental performance: a proposed model. Int. J. Econ. Financ. 9 (8), 118.

Arts, J., Runhaar, H.A., Fischer, T.B., Jha-Thakur, U., van Laerhoven, F., Driessen, P.P., Onyango, V., 2012. The effectiveness of EIA as an instrument for environmental governance: reflecting on 25 years of EIA practice in the Netherlands and the UK. J. Environ. Assess. Policy Manag. 14 (4), 1250025.

Berke, P.R., Conroy, M.M., 2000. Are we planning for sustainable development? An evaluation of 30 comprehensive plans. J. Am. Plann. Assoc. 66 (1), 21-33.

Bottrill, M.C., Pressey, R.L., 2012. The effectiveness and evaluation of conservation planning. Conserv. Lett. 5 (6), 407-420.

Bryson, J.M., 2018. Strategic Planning for Public and Non-profit Organizations: a Guide to Strengthening and Sustaining Organizational Achievement. John Wiley \& Sons.

Bryson, J.M., Alston, F., 2004. Creating and Implementing Your Strategic Plan, 2nd ed. JosseyBass, San Francisco.

de Montis, A., Ledda, A., Caschili, S., Ganciu, A., Barra, M., 2014. SEA effectiveness for landscape and master planning: an investigation in Sardinia. Environ. Impact Assess. Rev. 47, $1-13$.

Dobrovolski, R., Diniz-Filho, J.A.F., Loyola, R.D., Júnior, P.D.M., 2011. Agricultural expansion and the fate of global conservation priorities. Biodivers. Conserv. 20, 2445-2459.

Drechsler, M., Eppink, F.V., Wätzold, F., 2011. Does proactive biodiversity conservation save costs? Biodivers. Conserv. 20, 1045-1055.

Fernandes, J.P., Guiomar, N., 2016. Environmental ethics: driving factors beneath behavior, discourse and decision-making. J. Agric. Environ. Ethics 29 (3), 507-540.

Fernandes, J.P., Freire, M., Guiomar, N., Gil, A., 2017. Using modeling tools for implementing feasible land use and nature conservation governance systems in small islands-the Pico Island (Azores) case-study. J. Environ. Manage. 189, 1-13.

Fernandes, J.P., Guiomar, N., Gil, A., 2018. Identifying key factors, actors and relevant scales in landscape and conservation planning, management and decision making: promoting effective citizen involvement. J. Nat. Conserv.

Gillespie, A., 2007. PESTEL Analysis of the Macro-environment. Foundations of Economics. Oxford University Press, USA.

Goodland, R., 2002. Sustainability: human, social, economic and environmental. Encyclopedia Glob. Environ. Change 5, 481-491.

Gregory, R., Failing, L., Harstone, M., Long, G., McDaniels, T., Ohlson, D., 2012. Structured Decision Making: a Practical Guide to Environmental Management Choices. John Wiley \& Sons.

Hayes, S.J., Barker, A., Jones, C.E., 2017. Re-examining the rationale for strategic assessment: an evaluation of purpose in two systems. J. Environ. Assess. Policy Manage. 19, 1750020.

Hellriegel, D., Jackson, S.E., Slocum Jr, J.W., 1998. Management, eighth edition. SouthWestern Publishing.

Hermoso, V., Clavero, M., Villero, D., Brotons, L., 2017. EU's conservation efforts need more strategic investment to meet continental commitments. Conserv. Lett. 10 (2), 231-237.

Hill, T., Westbrook, R., 1997. SWOT analysis: it's time for a product recall. Long Range Plann. 30 (1), 46-52.

Izakovičová, Z., Špulerová, J., Petrovič, F., 2018. Integrated approach to sustainable land use management. Environments 5 (3), 37.

Johnson, G., Scholes, K., Whittington, R., 2008. Exploring Corporate Strategy: Text \& Cases. Pearson Education.

Kaplan, R.S., 2009. Conceptual foundations of the balanced scorecard. Handb. Manag. Account. Res. 3, 1253-1269.

Kaplan, R., 2012. The balanced scorecard: comments on balanced scorecard commentaries. J. Account. Organ. Chang. 8 (4), 539-545.

Kaplan, R.S., Norton, D.P., 2001. Transforming the balanced scorecard from performance measurement to strategic management: part I. Account. Horiz. 15 (1), 87-104.

Kaplan, R.S., Kaplan, R.E., Norton, D.P., Norton, D.P., Davenport, T.H., 2004. Strategy Maps: Converting Intangible Assets Into Tangible Outcomes. Harvard Business Press.

Laurian, L., Day, M., Backhurst, M., Berke, P., Ericksen, N., Crawford, J., Dixson, J., Chapman, S., 2004. What drives plan implementation? Plans, planning agencies and developers. J. Environ. Plan. Manage. 47 (4), 555-577.

Linnenluecke, M.K., Griffiths, A., 2010. Corporate sustainability and organizational culture. J. World Bus. 45 (4), 357-366.

Lobos, V., Partidario, M., 2014. Theory versus practice in strategic environmental assessment (SEA). Environ. Impact Assess. Rev. 48, 34-46.

Maon, F., Lindgreen, A., Swaen, V., 2010. "Organizational stages and cultural phases: a critical review and a consolidative model of corporate social responsibility development". Int. J. Manag. Rev. 12 (1), 20-38 ISSN 1460-8545.

Mickwitz, P., 2003. A framewsork for evaluating environmental policy instruments: context and key concepts. Evaluation 9 (4), 415-436.

Milieu, IEEP, ICF, 2016. Evaluation Study to Support the Fitness Check of the Birds and Habitats Directives. Milieu. Brussels.

Moore, J.L., Runge, M.C., 2012. Combining structured decision making and value-of-information analyses to identify robust management strategies. Conserv. Biol. 26 (5), 810-820.

Niven, P., 2010. Balanced Scorecard Step-by-Step. John Wiley \& Sons, Inc, Hoboken.

Parmenter, D., 2015. Key Performance Indicators: Developing, Implementing, and Using Winning KPIs. John Wiley \& Sons.

Pinto-Correia, T., Azeda, C., 2017. Public policies creating tensions in Montado management models: insights from farmers' representations. Land Use Policy 64, 76-82.

Sandman, P.M., 1993. Responding to Community Outrage: Strategies for Effective Risk Communication. AIHA.

Schäffer, U., Willauer, B., 2002. Kontrolle, Effektivität der Planung und Erfolg von Geschäftseinheiten-Ergebnisse einer empirischen Erhebung. Zeitschrift für Planung 13 (1), 73-97.

Soma, K., Onwezen, M.C., Salverda, I.E., van Dam, R.I., 2016. Roles of citizens in environmenta governance in the Information Age - four theoretical perspectives. Curr. Opin. Environ. Sustain. 18, 122-130.

Tippett, J., Searle, B., Pahl-Wostl, C., Rees, Y., 2005. Social learning in public participation in river basin management - early findings from Harmoni COP European case studies. Environ. Sci. Policy 8, 287-299. 